\title{
Phytosulfokine Is Involved in Positive Regulation of Lotus japonicus Nodulation
}

\author{
Chao Wang, ${ }^{1,2,3}$ Haixiang $\mathrm{Yu},{ }^{2}$ Zhongming Zhang, ${ }^{2}$ Liangliang $\mathrm{Yu},{ }^{1} \mathrm{Xiaoshu} \mathrm{Xu},{ }^{3}$ Zonglie Hong, ${ }^{4}$ \\ and Li Luo ${ }^{1}$ \\ ${ }^{1}$ Shanghai Key Lab of Bio-energy Crops, School of Life Sciences, Shanghai University, Shanghai, 200444, China; ${ }^{2}$ State Key \\ Laboratory of Agricultural Microbiology, Huazhong Agricultural University, Wuhan 430070, China; ${ }^{3}$ State Key Lab of Plant \\ Molecular Genetics, Institute of Plant Physiology and Ecology, Shanghai Institutes for Biological Sciences, Chinese Academy of \\ Sciences, Shanghai, 200032, China; ${ }^{4}$ Department of Plant, Soil, and Entomological Sciences and Program of Microbiology, \\ Molecular Biology and Biochemistry, University of Idaho, Moscow, ID 83844, U.S.A.
}

Submitted 9 March 2015. Accepted 9 March 2015.

Phytosulfokine (PSK) is a tyrosine-sulfated peptide that is widely distributed in plants, participating in cell proliferation, differentiation, and innate immunity. The potential role of PSK in nodulation in legumes has not been reported. In this work, five PSK precursor genes were identified in Lotus japonicas, designated as LjPSK1 to LjPSK5. Three of them (LjPSK1, LjPSK4, and LjPSK5) were found to be expressed in nitrogenfixing root nodules. LjPSKI and LjPSK4 were not induced at the early stage of nodulation. Interestingly, while the expression of LjPSK4 was also found in spontaneous nodules without rhizobial colonization, LjPSK1 was not induced in these pseudo nodules. Promoter- $\beta$-glucuronidase analysis revealed that LjPSK1 was highly expressed in enlarged symbiotic cells of nodules. Exogenous addition of $1 \mu M$ synthetic PSK peptide resulted in increased nodule numbers per plant. Consistently, the number of mature nodules but not the events of rhizobial infection and nodule initiation was increased by overexpressing LjPSK1 in transgenic hairy roots, in which the expression of jasmonate-responsive genes was found to be repressed. These results suggest that PSK is a new peptide signal that regulates nodulation in legumes, probably through cross-talking with other phytohormones.

Legumes interact with soil bacteria rhizobia and form a new root organ known as the nodule, in which rhizobia fix atmospheric dinitrogen into reduced nitrogen compounds that are useable by plants. Nodulation (Nod) factors are the most important signal molecule secreted by rhizobia during establishment of the Rhizobium-legume symbiosis (Downie 2014; Oldroyd and Downie 2008). At the early stage of symbiosis, the roots of legumes exude specific secondary metabolites, such as flavonoids, that are capable of entering rhizobia and inducing the expression of nodulation genes responsible for Nod factor biosynthesis. Rhizobial Nod factors are lipo-chitooligosaccharides

Nucleotide sequence data are available in the GenBank database under accession numbers KM065832 for LjPSK1, KM065833 for LjPSK2, KM065834 for LjPSK3, KM065835 for LjPSK4, and KM065836 for LjPSK5.

Corresponding author: Li Luo; Telephone: +86-21-66135321; Fax: +86-2166135321; E-mail: liluo@shu.edu.cn

*The $\boldsymbol{e}$-Xtra logo stands for "electronic extra" and indicates that six supplementary figures are published online.

(c) 2015 The American Phytopathological Society that are recognized by two lysin-motif (LysM) receptor kinases (NFR1 and NFR5 in Lotus japonicus or NFP and LYK3 in Medicago truncatula). After perception of the Nod factor signal, the receptors elicit a specific signal cascade, resulting in the reprogrammed expression of downstream genes, such as NIN and ENOD40 (Fang and Hirsch 1998; Limpens et al. 2003; Madsen et al. 2003; Radutoiu et al. 2003; Smit et al. 2007). Susceptive root systems allow rhizobia to enter epidermal cells through the development of infection threads (IT). Subsequently, rhizobia are released from the end of IT into de-differentiated host cortical cells. Functional nitrogen-fixing nodules are formed when infected rhizobia undergo differentiation into bacteroids that are enclosed by a plant-derived membrane known as the peribacteroid membrane. Root nodules or nodule-like structures can also be induced spontaneously, which can develop in the absence of rhizobia in legume roots with gain-of-function mutations in $\mathrm{Ca}^{2+} /$ calmodulindependent protein kinase (CCaMK) or cytokinin histidine kinase receptor (Gleason et al. 2006; Tirichine et al. 2006). Spontaneous nodules exhibit cortical cell division as the wild-type nodules do but do not contain rhizobia and, thus, do not fix atmospheric nitrogen.

The number of nodules developed on a root system is strictly regulated through a complex root-to-shoot-to-root negative feedback loop, known as autoregulation of nodulation (Ferguson et al. 2010). Root-derived signals are transported to the shoot, in which the signals are perceived by a leucine-rich repeat receptor kinase (LjHAR1, MtSUNN, and GmNARK) (Krusell et al. 2002; Schnabel et al. 2005; Searle et al. 2003). Null mutations of these genes result in deregulated hypernodulation after inoculation with rhizobia. Recently, root-derived CLAVATA3/ESRrelated (CLE) glycopeptides from $L$. japonicus have been found to serve as the ligands of LjHAR1 and to be capable of suppressing nodulation (Okamoto et al. 2013). CLE are small, secreted peptides that universally exist in plant species, including legumes. At least 39, 25, and $39 C L E$ genes have been identified in the genomes of L. japonicus, Medicago truncatula, and Glycine max, respectively (Funayama-Noguchi et al. 2011; Mortier et al. 2010, 2011). In L. japonicus, LjCLE-RS1 and $L j C L E-R S 2$ are strongly upregulated in roots that have been inoculated with rhizobia. Overexpression of either of $L j C L E-R S 1$ and $L j C L E-R S 12$ genes in hairy roots can result in a systemic inhibition of nodulation in a HAR1-dependent manner (Okamoto et al. 2009). Conversely, knockdown of MtCLE12 and MtCLE13 increases the number of nodules per root system (Mortier et al. 2012). Functionally similar CLE genes have also been found in Glycine max (Reid et al. 2011). 
Other plant peptides have also been implicated in nodule development. The putative peptide products of ENOD4O are required for the differentiation of root cortical cells and the initiation of nodule primordia. The expression of ENOD4O genes is induced in roots at the very early stage of rhizobial infection or Nod factor application. Knockdown of ENOD40 gene expression leads to significant suppression of nodule formation but has no effect on the early bacterial infection events, indicating a growth-regulating role of ENOD40 in nodule organogenesis (Kumagai et al. 2006; Wan et al. 2007). Recently, Medicago truncatula C-terminally encoded peptide 1 (MtCEP1) has been shown to act as a developmental signal to enhance nodulation. Overexpression of MtCEPI or application of the MtCEP1 peptide to roots results in inhibition of lateral root formation and enhancement of nodulation (Imin et al. 2013). The molecular mechanism by which CEP1 regulates nodulation is not clear. Nodule-specific cysteine-rich peptides have recently been identified, and their role in bacteroid differentiation during symbiosis has been suggested (Farkas et al. 2014; Penterman et al. 2014; Van de Velde et al. 2010).

Phytosulfokine (PSK)- $\alpha$ represents the first sulfated peptide $\left(\mathrm{Y}_{\mathrm{SO} 3 \mathrm{H}} \mathrm{IY}_{\mathrm{SO} 3 \mathrm{H}} \mathrm{TQ}\right)$ identified in plants. PSK- $\alpha$ has been shown to regulate cell proliferation in cell cultures of Asparagus officinalis L. and rice (Oryza sativa L.) (Matsubayashi and Sakagami 1996; Matsubayashi et al. 1997). PSK- $\alpha$ also acts as a growth-promotion factor for hypocotyls, roots, and leaves (Huang et al. 2010; Kutschmar et al. 2009; Stührwohldt et al. 2011). PSK is derived from prepropeptides encoded by five PSK genes in Arabidopsis thaliana. It acts as a peptide hormone, whose signal is perceived by the Arabidopsis PSK receptor kinase 1 (AtPSKR1) (Matsubayashi et al. 2006), a leucine-rich repeat-containing receptor-like kinase. The PSK signal can also, to a minor extent, be perceived by AtPSKR2 (Kutschmar et al. 2009). PSK precursors are sulfated on two conserved tyrosine residues at their C-termini by a tyrosylprotein sulfotransferase (TPST) (Komori et al. 2009). The sulfated precursors are secreted into the extracellular space or apoplast after cleavage of their N-terminal signal sequence by signal peptidases. A subtilisin serine protease, AtSBT1.1, has been proposed to be required for the processing of AtPSK4 (Srivastava et al. 2008). PSK also participates in wounding responses and immune signal transduction during plant-microbe interactions. PSK signaling plays an opposite role in the immune response of Arabidopsis to biotrophic and necrotrophic pathogens. This antagonistic manner appears to depend on modulated homeostasis between the salicylate and jasmonate signaling pathways (Igarashi et al. 2012; Mosher et al. 2013). Homologous genes of PSK are widely present in higher plants including legumes, but whether PSK regulates legume nodulation is not known.

In this work, we identified five PSK precursor genes in L. japonicus and characterized their expression patterns. The potential functions of a nodule-specific PSK precursor gene, LjPSK1, and a chemically synthesized PSK peptide on nodulation were examined. Our results suggest that PSK may positively regulate legume nodulation in a manner independent of the Nod factor signaling pathway.

\section{RESULTS}

Identification of PSK precursor genes in L.japonicus.

To identify PSK precursor genes in L. japonicus, Arabidopsis PSK precursors were used as queries to search all available L. japonicus databases by BLAST. Two genes, annotated as chr5. CM0359.140.r2 and chr2.CM0124.210.r2/partial, were found in the $L$. japonicus genome. Further search of the expressed sequence tag database using the C-terminal regions of chr5.CM0359.140.r2 and chr2.CM0124.210.r2/partial led to identification of five putative PSK-encoding sequences, including GO020953.1, FS350221.1, GO018273.1, GO036043.1, and GO036835.1. Among them, GO020953.1 and FS350221.1 are identical to chr5.CM0359.140.r2 and chr2.CM0124.210.r2, respectively. These PSK-encoding sequences were amplified by polymerase chain reaction (PCR), were confirmed by DNA sequencing, and were named LjPSK1 to LjPSK5. Alignment of deduced amino acid sequences of LjPSK1 to LjPSK5 with AtPSK1 to AtPSK5 showed that all PSK precursors contain a cleavable secretory signal sequence at the $\mathrm{N}$-termini and an active peptide sequence close to the $\mathrm{C}$ termini, where conserved amino acids (Cys, two Arg or Lys, His, and Asp) can be identified (Fig. 1A). Analysis of the amplified genomic DNAs of LjPSK1 to LjPSK5 showed that LjPSK genes contain two to three exons (Fig. 1B), which is similar to AtPSK and OsPSK genes (Yang et al. 2000, 2001).
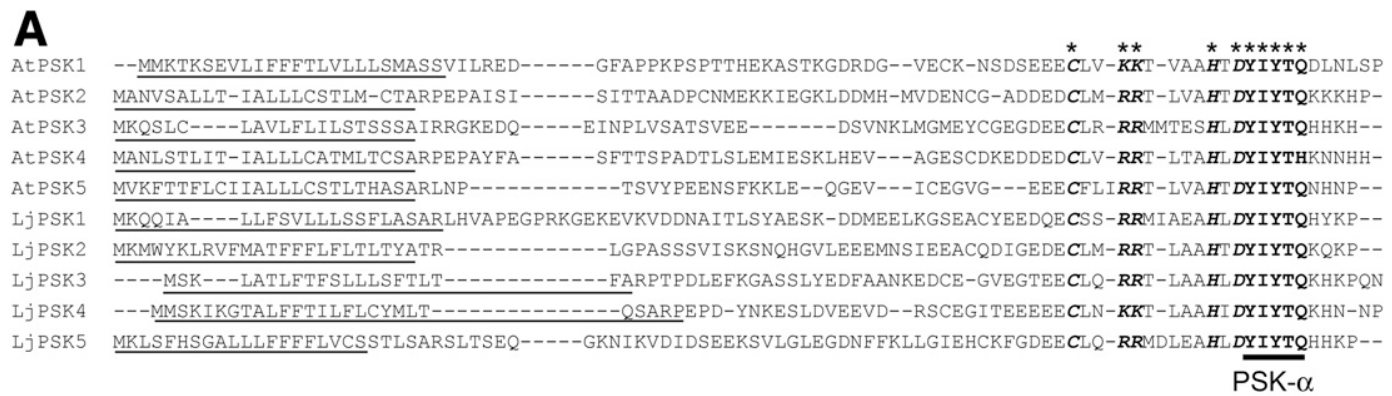

B

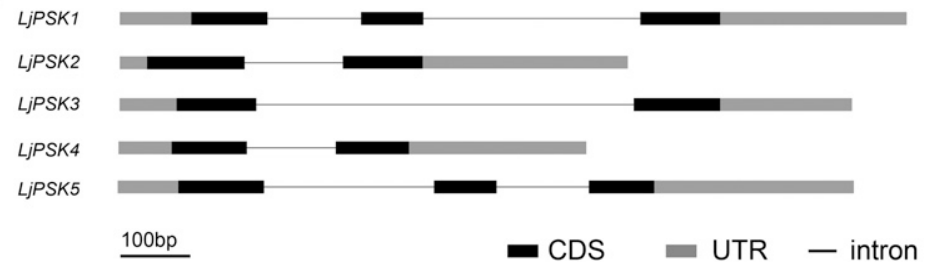

Fig. 1. Amino acid sequence alignment and gene structure of the phytosulfokine (PSK) gene family in Lotus japonicus. A, Amino acid sequences of the PSK gene family from L. japonicus and Arabidopsis thaliana were aligned using ClustalX. The conserved residues were marked with asterisks. The predicted secretory signal peptides were underlined. B, Gene structures of LjPSK1 to LjPSK5 were predicted, using the mRNA-to-genomic alignment program Spidey. LjPSK contains one to two introns that are indicated by lines. Black and gray boxes represent the coding sequences (CDS) and untranslated regions (UTR), respectively. 


\section{Expression profiles of $L j P S K$ genes.}

To characterize the expression profiles of LjPSK genes, we evaluated the transcript abundance by quantitative reverse transcriptional real-time (qRT)-PCR in roots, stems, leaves, flowers, nodules and pods (Fig. 2A). Transcripts of LjPSK genes were detected in all tissues tested, but certain LjPSK genes were predominantly expressed in one or two tissues. For example, LjPSK1 and LjPSK4 were expressed at much higher levels in nodules than in other tissues. The LjPSK3 mRNA was most detected in stems and flowers. LjPSK5 was found mostly in roots, nodules, and pods. Analysis of the transcriptomic data in the other legume model plant Medicago truncatula reveals that MtPSK genes are highly expressed in nodules, implying a conserved role of PSK in nodulation (Supplementary Fig. S1)
(Roux et al. 2014). The maturation process of a PSK peptide includes sulfation of the peptide by a TPST, encoded by AtTPST in Arabidopsis. In Medicago, a putative MtTPST is constitutively expressed in both roots and nodules, with an elevated level in the latter. We also identified the putative ortholog of AtTPST in L. japonicus (LjSGA_033116.1). Expression analysis by qRT-PCR revealed that LjTPST is expressed at a higher level in nodules than roots, which is consistent with the expression pattern of MtTPST in Medicago spp. (Supplementary Fig. S2).

\section{Specific expression of LjPSK1 and LjPSK4 in nodules.}

Nodules at different development stages were collected for qRT-PCR analysis of the expression of LjPSK1 and LjPSK4.
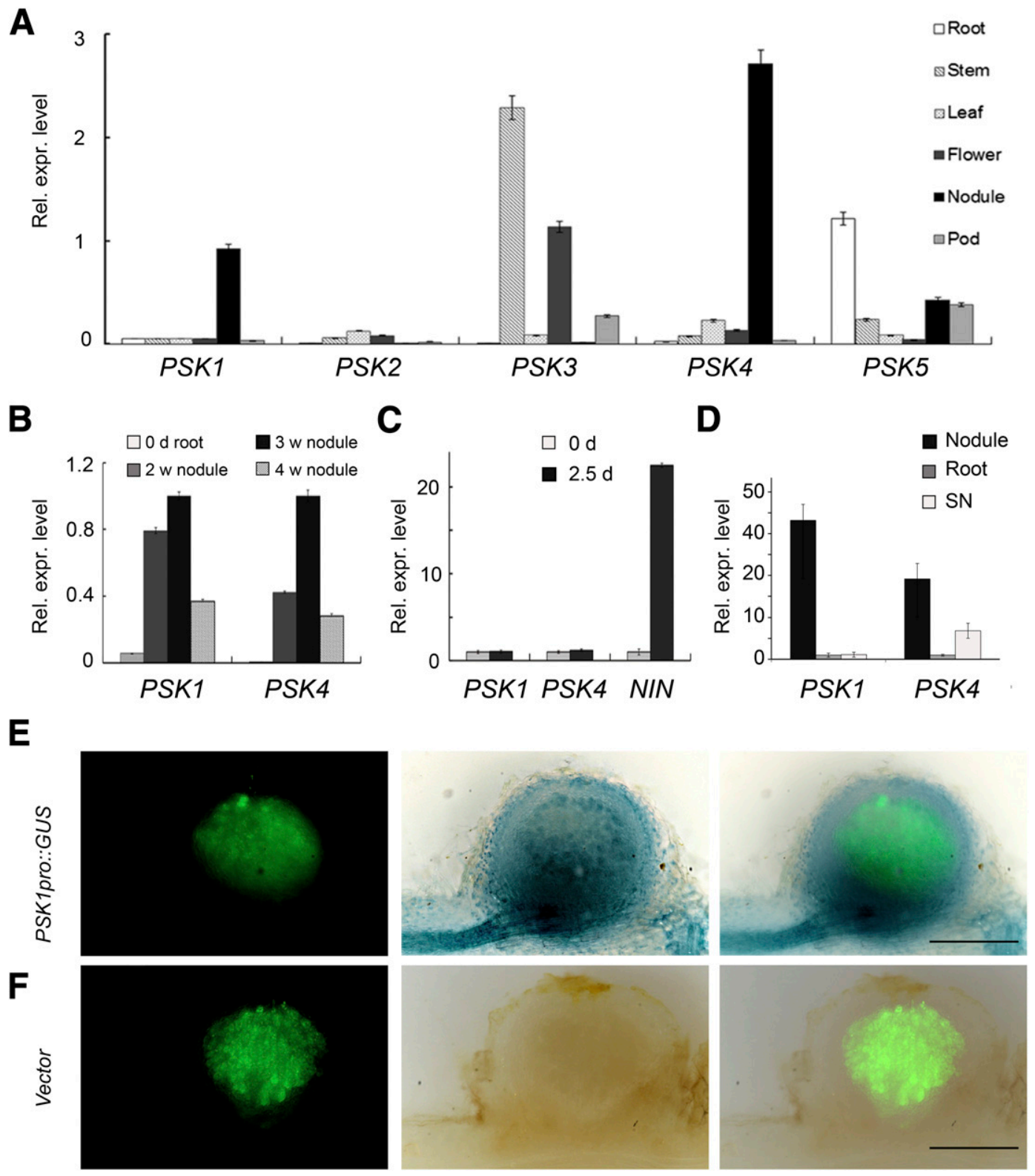

Fig. 2. Expression profiles of LjPSK1 to LjPSK5. A, Expression patterns of LjPSK1 to LjPSK5 genes assessed by quantitative reverse transcription-polymerase chain reaction. Total RNA was isolated from Lotus japonicus uninoculated roots, 3-week-old stems, leaves, nodules, flowers, and pods. Relative expression levels (Rel. expr. level) were normalized against the internal control of ATPase gene expression. B, RNA samples were obtained from nodules 2 to 4 weeks after inoculation with Mesorhizobium loti. Uninoculated roots served as a control. C, Expression of LjPSK1 and LjPSK4 in roots 2.5 days postinoculation with $M$. loti, as compared with the uninoculated roots $(0 \mathrm{~d})$. The expression of NIN was used as a marker gene to indicate normal response of an early nodulation gene to rhizobial infection. D, Expression of LjPSK1 and LjPSK4 in 3-week-old wild-type nodules, nodule-stripped roots, and spontaneous nodules produced on transgenic hairy roots expressing $L j C C a M K^{T 265 D}$ on $n f r 5-2$ mutant plants. Error bars indicate \pm standard error from three biological replicates. E, Transgenic hairy roots expressing LjPSK1 promoter- $\beta$-glucuronidase (GUS) fusion were inoculated with $M$. loti constitutively expressing GFP and nodules were stained for $8 \mathrm{~h}$ for GUS activity analysis. F, Nodules on transgenic hairy roots expressing an empty vector served as a negative control. 
The results showed that the expression of both genes peaked in nodules 3 weeks postinoculation with Mesorhizobium loti and decreased drastically in 4-week-old nodules, suggesting that PSK is involved in nodule development and may not be required for nitrogen fixation in mature nodules (Fig. 2B). At the early stage of nodulation, the expression of LjPSK1 and LjPSK4 did not appear to be induced by inoculation with $M$. loti (Fig. 2C) as compared with the positive control gene LjNIN, which showed normal induction by rhizobial inoculation (Fig. 2C). Similar samples were used to test the expression level of LjTPST, and no induction of LjTPST at the early stage of nodulation was observed.

In nodules, the expression pattern of LjPSK1 and LjPSK4 was further investigated in spontaneous nodules in comparison with the wild-type nodules and nodule-stripped roots 3 weeks postinoculation with $M$. loti. To produce spontaneous nodules, we used Agrobacterium rhizogenes-mediated transformation to generate chimeric transgenic plants with transformed hairy roots attached to an untransformed shoot (Kumagai and Kouchi 2003). Spontaneous nodules were developed on transgenic hairy roots constitutively expressing the gain-of-function mutation of $L j C C a M K, L j C C a M K^{T 265 D}$, under the control of a ubiquitin promoter. The nfr5-2 mutant plants were used to produce transgenic hairy roots. As shown in Figure 2D, the expression level of the nodule-specific $L j P S K 1$ in spontaneous nodules was similar to that in roots. However, the expression level of LjPSK4 was elevated in spontaneous nodules. The results suggest that the

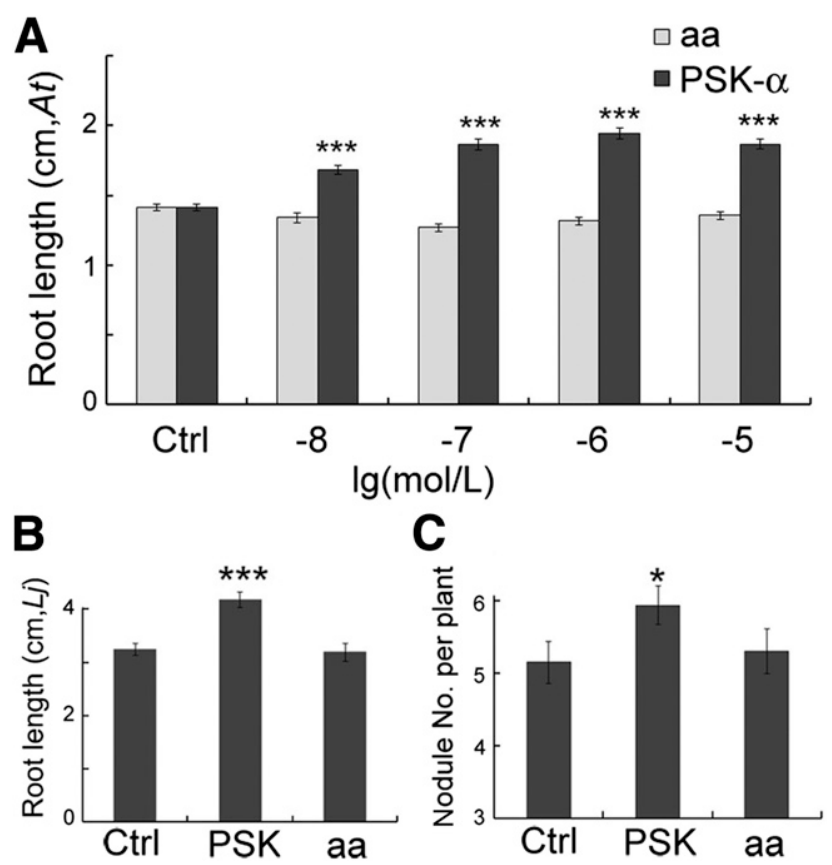

Fig. 3. Effects of phytosulfokine (PSK)- $\alpha$ peptide on primary root growth and nodulation of Lotus japonicus. A, Sterilized Arabidopsis seeds were grown on half-strength Murashige Skoog (MS) medium containing $10^{-8}$ to $10^{-5} \mathrm{M}$ of PSK- $\alpha$ peptide. Root length (mean \pm standard error [SE]) was determined 6 days after seed germination from 50 to 60 plants per treatment. Treatment with a mixture of the amino acids (aa) comprising PSK served as a control, showing no enhanced root growth. Plants grown on medium without PSK treatments were used as an untreated control (Ctrl). B, L. japonicus seedlings were transferred onto half-strength MS plates containing $1 \mu \mathrm{M}$ PSK- $\alpha$ and were grown for 6 days. Root length was determined (mean \pm SE) with 25 to 40 plants. C, L. japonicus seedlings were transplanted to growth pots, were inoculated with Mesorhizobium loti, and were treated with $1 \mu \mathrm{M}$ PSK- $\alpha$. Nodules per plant were counted from more than 35 plants 12 days after inoculation with $M$. loti. Values that are significantly different from the control, as determined by Student's $t$ test, are marked with one $(P<0.05)$ or three $(P<0.001)$ asterisks. induced expression of LjPSK1 but not LjPSK4 in wild-type nodules depends on rhizobial colonization in nodules.

\section{Promoter- $\beta$-glucuronidase (GUS) analysis of LjPSK1 in nodules.}

To study the expression pattern of LjPSK1 at a cellular level in nodules, an approximate 4-kb promoter fragment upstream of the start codon was cloned and was used to drive the expression of the GUS reporter gene. The harl hypernodulation mutant plants were used to produce transgenic hairy roots, which were found to be able to develop more nodules. GUS-staining analysis showed high expression of LjPSK1 in the whole nodule, including the central zone and surrounding cortical cells (Fig. 2E). Interestingly, at higher magnifications, higher GUS activity was observed in Rhizobium-infected cells than uninfected cells in the central zone of a nodule (Fig. 2E). Nodules formed on hairy roots expressing an empty vector served as a control, which showed no GUS activity (Fig. 2F).

\section{Promotion of $L$. japonicus root growth and nodule formation by synthetic PSK- $\alpha$.}

PSK- $\alpha$ has been reported to function as a growth factor to modulate cell proliferation and differentiation. At a concentration range between $10 \mathrm{nM}$ and $10 \mu \mathrm{M}$, synthetic PSK- $\alpha$ peptide promoted $A$. thaliana root growth (Fig. 3A), which is consistent with the observation described previously (Kutschmar et al. 2009). The length of $A$. thaliana roots increased as the concentration of PSK- $\alpha$ in the growth medium was elevated to approximately $1 \mu \mathrm{M}$ (Fig. 3A). L. japonicus roots were significantly elongated from 3.24 to $4.17 \mathrm{~cm}$ after 6 days of growth on plates containing $1 \mu \mathrm{M}$ PSK- $\alpha$ (Fig. 3B), suggesting that a common PSK- $\alpha$ response system exists in A. thaliana and $L$. japonicus. To estimate a possible effect of PSK- $\alpha$ on nodulation, L. japonicus plants were supplied with nitrogen-free liquid medium containing both rhizobia and $1 \mu \mathrm{M}$ PSK- $\alpha$. The nodule number per root system, as counted 12 days after rhizobial inoculation, was higher in roots treated with PSK- $\alpha$ than in the control roots that were either untreated with PSK- $\alpha$ or treated with a mixture of the free amino acids constituting PSK (Fig. 3C). The promotion effect of PSK- $\alpha$ on nodulation was relatively weak, yet statistically significant. These observations indicate that PSK- $\alpha$ could positively regulate primary root growth and nodulation in L. japonicus.

\section{Promotion of nodulation on transgenic hairy roots overexpressing LjPSK1 or AtPSKR1.}

To confirm the role of PSK signaling in nodulation, the L. japonicus PSK- $\alpha$ precursor gene LjPSK1 and A. thaliana PSK- $\alpha$ receptor gene AtPSKRl were overexpressed in $L$. japonicus hairy roots under the control of an enhanced Cauliflower mosaic virus (CaMV) 35S or a ubiquitin promoter, respectively. The transcript levels of LjPSK1 and AtPSKR1 were determined by RT-PCR, confirming that they were overexpressed in transgenic roots as compared with the roots transformed by the empty vector, pC2301, or pUBGFP (Fig. 4C and D). The numbers of white bumps and yellow and red nodules, corresponding to the nodule primordia and developing and mature nodules, respectively, were recorded 12 days postinoculation with $M$. loti. The results show that the nodule numbers per plant, particularly the developing and mature ones, were significantly greater on the hairy roots overexpressing LjPSK1 and AtPSKR1 than those on the controls (Fig. 4A and B). This experiment was repeated three times and similar results were obtained, suggesting that the PSK- $\alpha$ peptide and PSK signaling play a positive role in regulation of nodule development. It is possible that the increased nodule number may arise as an accessary consequence of the enhanced hairy-root growth by PSK signaling, because treatment of PSK- $\alpha$ 
peptide was able to promote the primary root growth (Fig. 3B). However, it is more likely that PSK- $\alpha$ signaling has a primary effect on nodulation, because enhanced nodulation on the hairy roots expressing either LjPSK1 or AtPSKRl could be easily observed as compared with the control hairy roots expressing an empty vector and there was no significant alteration between the two hairy-root samples (Supplementary Fig. S3).

\section{Enhancement of the PSK- $\alpha$ signaling in Arabidopsis plants overexpressing LjPSK1.}

We further examined whether overexpression of $L$. japonicus PSK1 in Arabidopsis would have any effect on vegetative growth similar to that triggered by overexpression of AtPSKR1. For this purpose, transgenic Arabidopsis plants expressing LjPSK1 or AtPSKR1 were generated using the same constructs described above. Homozygous T3 transgenic seeds were obtained and two independent lines for LjPSK1-OX (overexpressing) or AtPSKR1-OX were used for phenotype analysis. Similar to the observation of AtPSKRI overexpression (Hartmann et al. 2013; Kutschmar et al. 2009; Matsubayashi et al. 2006), Arabidopsis transgenic plants overexpressing $L j P S K 1$ exhibited phenotypes of leaf enlargement, root elongation (Fig. 4E, F, and G), and enhanced plant growth (Supplementary Fig. S4). Analysis of qRT-PCR revealed an eightfold increase in the AtPSKR1 mRNA level in the AtPSKR1-OX line over the control plant (Fig. 4H). While the LjPSK1 transcript could not be detected in the wild-type Arabidopsis plants, a high level of the LjPSK1 mRNA could be detected in the LjPSK1-OX line (Fig. 4H). We conclude that overexpression of LjPSK1 or AtPSKRI in transgenic Arabidopsis plants can lead to enhanced PSK- $\alpha$ signal transduction, promoting vegetative growth.

\section{No observable effect of PSK signaling on rhizobial} infection and nodule initiation.

To determine whether PSK signaling modulates L. japonicus nodulation at the early stage of Rhizobium-legume symbiosis, we examined rhizobial infection and nodule initiation on transgenic hairy roots overexpressing LjPSK1 or AtPSKR1. Microscopic observations showed that the total rhizobial infection events, including numbers of infection foci and IT, were not significantly different between transgenic roots overexpressing LjPSK1 or AtPSKRI and the controls expressing the corresponding empty vectors. The numbers of nodule primordia per plant on transgenic roots were also not significantly changed. We further analyzed the expression of early nodulation genes NIN and ENOD40.1 in the transgenic roots overexpressing AtPSKRI and found that the abundance of NIN and ENOD40.1 transcripts was not significantly altered in transgenic roots as compared with those transformed by the empty vector. These results suggest that the effect of PSK on nodulation may not be associated with the rhizobial infection and nodule primordium initiation in the early stage of nodulation (Supplementary Fig. S5).

\section{Repression of jasmonate-responsive genes by overexpression of LjPSK1 or AtPSKR1.}

In Arabidopsis, PSK signaling has been shown to regulate jasmonic acid (JA)-mediated plant immunity. JA has been reported as a negative regulator for nodulation of legumes with both determinate and indeterminate nodules, such as $L$. japonicus and Medicago truncatula (Nakagawa and Kawaguchi 2006; Sun et al. 2006). To test if the effect of PSK on L. japonicus
A
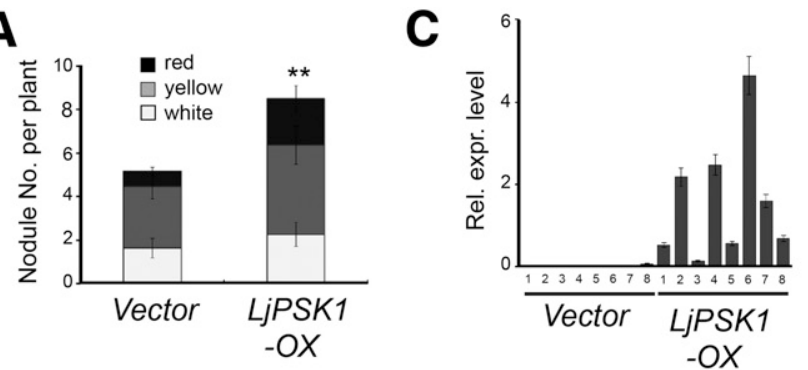

B

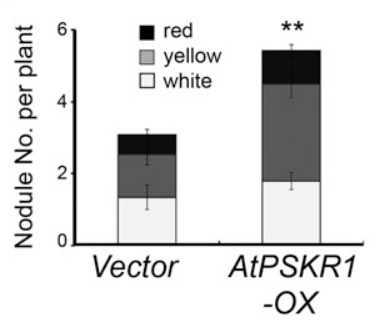

D

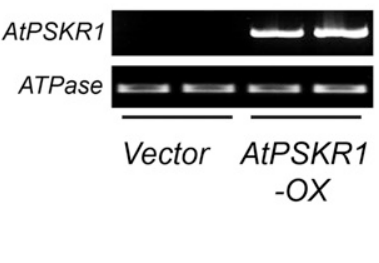

E

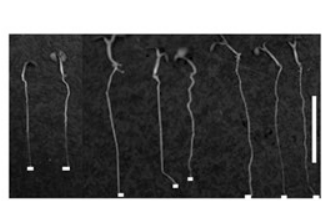

Col-0 LjPSK1 AtPSKR1
G

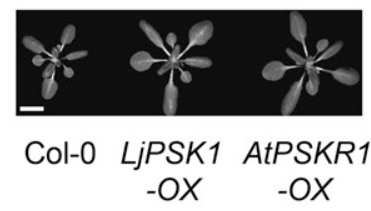

$\mathbf{F}$

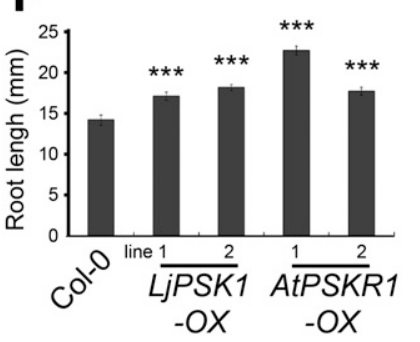

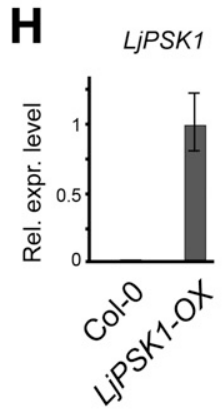

AtPSKR1

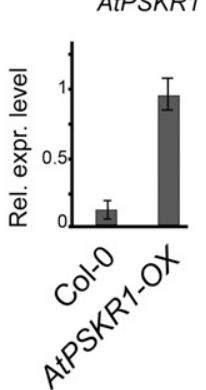

Fig. 4. Effects of LjPSK1 or AtPSKR1 overexpression on nodulation and plant growth. Transgenic hairy roots of Lotus japonicus expressing LjPSK1 or AtPSKR1 were generated via Agrobacterium rhizogenes-mediated transformation. Transgenic plants expressing the empty vectors (pC2301 for LjPSK1-OX and pUBGFP for AtPSKR1-OX) served as controls. Transgenic Arabidopsis plants were obtained and T3 homozygous seeds were used for assays. Two representative lines from each group were characterized and compared with wild-type (Col-0) plants. Values in A, B, and F represent the mean \pm standard error (SE) of 15 to 30 plants. A, The nodule numbers per plant were increased significantly $(P<0.01)$ in hairy roots expressing $L j P S K 1$-OX $(n=21)$ as compared with the control plants $(n=18) 12$ days postinoculation with Mesorhizobium loti. Two additional replications were performed with a total of 51 plants for the control and 65 plants for LjPSK1-OX lines, producing a similar result. Nodule primordia (white), young nodules (gray) and mature nodules (black) were recorded separately. B, Hairy roots expressing AtPSKR1-OX $(n=53)$ formed more nodules as compared with the control $(n=26)$. Statistical significant difference is indicated by two asterisks $(P<0.01)$. C, Quantitative and reverse transcription-polymerase chain reaction (RT-PCR) and D, RT-PCR were used to assess the expression levels of LjPSK1 and AtPSKR1 in hairy roots. E, Arabidopsis plants grown on half-strength Murashige Skoog (MS) medium for 6 days. Arabidopsis root elongation was enhanced significantly by LjPSK1-OX or AtPSKR1-OX. F, Statistical analysis of primary root length (mean \pm SE) of seedlings grown on half-strength MS medium for 6 days. G, Arabidopsis plants grew in growth cabinets for 2 weeks. Leaf growth was enhanced by expressing LjPSK1-OX or AtPSKR1-OX. H, Expression levels of the LjPSK1 and AtPSKR1 transcripts in transgenic LjPSK1- and AtPSKR1-overexpression lines. Values represent the average levels $( \pm \mathrm{SE})$ of two transgenic lines of either LjSPK-OX or AtPSKR1-OX. Bars, $1 \mathrm{~cm}$. 
nodulation is correlated with suppression of JA signal transduction, we analyzed the expression of putative JA-responsive genes in three to eight independent transgenic root lines overexpressing LjPSK1 or AtPSKR1 in the absence of rhizobia. It has previously been reported that the $L$. japonicus genes encoding proteins homologous to PDF1.2, JAR1, and MYC2 are induced by JA (Suzuki et al. 2011). PDF1.2 is a defensin in the JAdependent defense response, JAR1 is an enzyme for the production of active JA derivative (e.g., JA-Ile), and MYC2 is a transcription activator of JA responses. Analysis of qRT-PCR results revealed that the expression of the three genes decreased significantly in transgenic roots overexpressing LjPSK1 or AtPSKR1 (Fig. 5) with the exception of JAR1, whose expression was not apparently changed in LjPSK1-OX roots (Fig. 5). These results suggest that the JA signal transduction pathway is downregulated by overexpression of LjPSK1 or AtPSKR1, which may explain why nodulation was increased in $L$. japonicus roots overexpressing LjPSK1 or AtPSKRI.

\section{DISCUSSION}

Recent studies have shown that tyrosine-sulfated peptides, including PSK, peptide containing sulfated tyrosine 1 (PSY1), and root-growth factor, are widely present in higher plants (Delay et al. 2013; Matsuzaki et al. 2010; Mosher et al. 2013). They function to control cell differentiation and organ development in plants. Legume root nodules are a unique nitrogenfixing organ composed of both the rhizobia and host cells. The initiation and development of nodules are tightly regulated by rhizobial Nod factors and plant hormones, including cytokinin, auxin, ethylene, jasmonate, and salicylic acid. PSK has been shown to regulate not only plant development but also plant innate immunity. In this work, we identified five L. japonicus PSK precursor genes (LjPSK1 to LjPSK5) and found that the expression of LjPSK1 and LjPSK4 were specific in nodules. Our

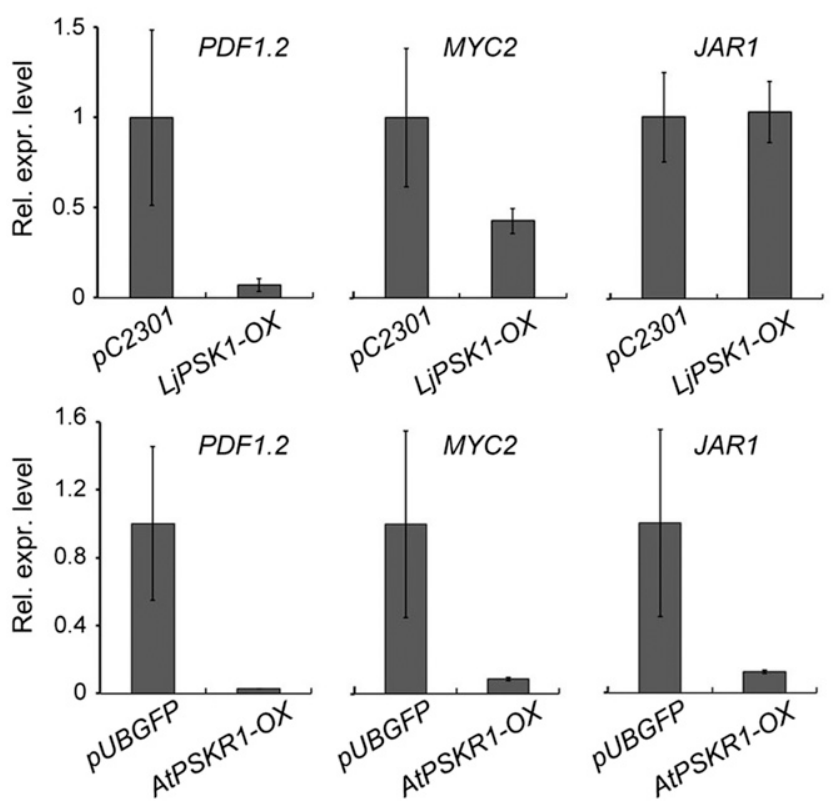

Fig. 5. Downregulation of jasmonic acid (JA)-responsive genes in transgenic roots. Total RNA was isolated from uninoculated hairy roots expressing LjPSK1-OX $(n=8)$, AtPSKR1-OX $(n=3)$, and the corresponding vector controls pC2301 $(n=3)$ and pUBGFP $(n=4)$. Quantitative reverse transcription-polymerase chain reaction was used to assay JA-responsive genes PDF1.2, MYC2, and JAR1. Values indicated the average expression levels of the individual hairy-root lines. Error bars indicate \pm standard deviation. findings show that PSK is a positive regulator for nodule development and may antagonize the JA signaling, which negatively regulates nodulation in a manner independent of the Nod factor pathway. Therefore, PSK may represent a new player in the regulatory cascade of nodule development.

\section{Search for a receptor of PSK in L. japonicus.}

Chemically synthesized PSK- $\alpha$ can activate PSK signaling through the receptor kinase AtPSKR1 to promote cell proliferation and plant growth (Kutschmar et al. 2009; Matsubayashi and Sakagami 1996; Matsubayashi et al. 1997; Stührwohldt et al. 2011). Our data confirmed a positive effect of synthesized PSK- $\alpha$ peptide on root growth in L. japonicus (Fig. 3B), suggesting that this peptide could be perceived by an unidentified LjPSK receptor, a possible ortholog of AtPSKR1. Our search of available databases using AtPSKR1 has not identified a gene encoding a putative LjPSK receptor. As the L. japonicus genome sequencing and annotation continues to be improved, an ortholog of AtPSKR1 may be identified in future studies.

\section{Role of PSK in nodule initiation, development, and maturation.}

The promotion effect of PSK- $\alpha$ on nodulation was relatively weak, despite being statistically significant (Fig. 3C). One of the explanations for this is that the active concentrations of PSK- $\alpha$ were difficult to be accurately maintained in pots in a greenhouse in the presence of rhizobia, because stability of the peptide could be affected by growth conditions. Because nodule primordia are initiated from cortical cell division, the perception of PSK by cortical cells is key to the function of PSK. Epidermal cells of roots might also be a natural barrier for the penetration of exogenous PSK. To overcome this, we generated $L$. japonicus transgenic hairy roots with enhanced endogenous PSK signaling by overexpression of the LjPSK1 precursor gene or AtPSKR1. More nodules per plant were produced in these transgenic roots, suggesting that PSK signaling was enhanced (Fig. 4). Nodule numbers per plant are a measurable trait contributed by the events of bacterial infection, number of IT, number of nodule primordia, and rate of nodule development. Our observations revealed that both processes of bacterial infection and nodule initiation did not seem to be affected significantly by overexpressing LjPSK1 or AtPSKR1. The lack of a role of LjPSK1 and LjPSK4 in early nodulation coincided with the low levels of LjPSK1 and LjPSK4 gene expression in the early stage of nodulation (Fig. 2C). Moreover, transgenic roots overexpressing $L j P S K 1$ or AtPSKR1 did not alter the expression of LjNIN and LjENOD40, two symbiotic genes downstream of the Nod factor signaling, suggesting that PSK signaling may not play a major role in the early stage of nodulation.

The increased nodule numbers per plant in transgenic roots expressing LjPSK1 and AtPSKRI (Fig. 4A and B) appear to result from an increased rate of nodule growth and maturation. We expressed LjPSK1 or AtPSKR1 in transgenic Arabidopsis plants and were able to confirm the function of LjPSK1 or AtPSKR1 overexpression in enhancing PSK signaling (Fig. 4E and F). In Arabidopsis, AtPSK2, AtPSK3, and AtPSK5 are more strongly expressed in lower mature leaves than in upper young leaves (Matsubayashi et al. 2006). PSK- $\alpha$ treatment promotes root growth by increasing meristem size and cell size (Kutschmar et al. 2009). Both reports imply PSK in the potential regulation of cellular growth rather than cell division and differentiation. It is possible that PSK- $\alpha$ may induce cell enlargement of $L$. japonicus nodules to promote nodule development and maturation.

\section{Redundancy of LjPSK genes.}

In Arabidopsis, five PSK precursor genes are expressed in all plant tissues with different and partly overlapping expression 
patterns (Kutschmar et al. 2009; Matsubayashi et al. 2006). AtPSK can also be induced by wounding and microbial interaction, indicating the specific response of PSK signaling to different stresses (Loivamäki et al. 2010; Matsubayashi et al. 2006). We found that LjPSK1, LjPSK4, and LjPSK5 were highly expressed in mature nodules in L. japonicus (Fig. 2A). The abundant levels of $L j P S K$ transcripts in developing nodules suggest that PSK signaling is involved in nodule growth and maturation. At the same time, the redundancy of $L j P S K$ genes in $L$. japonicus makes it difficult to study gene function through reverse genetics analysis such as RNA interference or LORE1 insertion mutants (Urbański et al. 2012). In this study, we identified L. japonicus pskl (30030761) and psk4 (30006132) mutant lines from the LORE1 mutant collections (Urbański et al. 2012). The homozygous plants of the two mutant lines exhibited normal growth and nodulation as compared with those in the wild-type L. japonicus. Although reduced nodulation was observed in the Ljpsk1 mutant line, the effect was very weak (Supplementary Fig. S6). To overcome the redundancy of LjPSK genes, double mutant pskl/psk4 plants should be generated through crossing of the two mutants. It remains to be tested if defective nodulation phenotypes can be observed in double mutant pskl/psk4 plants. LjPSK genes showed different tissuespecific expression patterns, except LjPSK2, which presented low expression levels in all tissues tested (Fig. 2). It is worth noting that refined regulation of $L j P S K$ was found in nodule cells, including infected and uninfected cells (Fig. 2D and E). Thus, PSK signaling plays a global role in plant growth, development, and reproduction, including nodule cell development.

\section{Cross-talks of PSK- $\alpha$ with JA signaling.}

PSK- $\alpha$ has been reported to modulate salicylic acid/JA hormone homeostasis to coordinate plant defense in A. thaliana (Mosher et al. 2013). JA is generally considered a defense hormone and a negative regulator on root-nodule formation in L. japonicus, although a positive role of JA on nodulation has also been reported (Suzuki et al. 2011). Most recent observations have indicated that JA biosynthesis and signaling genes are repressed by rhizobial inoculation in the early stage of nodule development (Breakspear et al. 2014). The downregulation of JA-responsive genes in L. japonicus transgenic roots expressing either LjPSK1 or AtPSKRI corresponded to the enhancement of nodulation (Fig. 5). Our results are consistent with the notion that JA may be a negative regulator of nodulation and suggest that PSK may antagonize JA signaling to control nodule development. It is also possible that PSK may suppress host defense responses in nitrogen-fixing nodules. During evolution, legumes might have hijacked a universal signaling pathway to regulate nodule organ development. In summary, the expression of two of the five LjPSK genes is highly enhanced during nodule development in L. japonicus, and PSK signals may be perceived by a receptor to control nodule growth and maturation.

\section{MATERIALS AND METHODS}

\section{Plant growth and bacterial strains.}

For nodulation assays, plants of L. japonicus MG-20 Miyakojima or Gifu B-129 were grown in a mixed soil medium containing perlite and vermiculite at a 1:1 volume ratio supplied with a half-strength $\mathrm{B} \& \mathrm{D}$ nitrogen-free medium in growth cabinets with a 16-h-light and 8-h-dark cycle at $22 \pm 1{ }^{\circ} \mathrm{C}$. Mesorhizobium loti MAFF303099 was used for inoculation of L. japonicus plants. The M. loti MAFF303099 strain that constitutively expresses a $l a c Z$ marker was used for rhizobial infection assays. A. thaliana plants were grown on soil supplied with a full-nutrition medium in a growth chamber with a 16-h-light and 8-h-dark cycle at $22 \pm 1^{\circ} \mathrm{C}$. A. rhizogenes LBA1334 was used for hairy-root transformation.
RNA isolation and gene expression assay.

For tissue-specific expression assays, total RNA from roots, stems, and leaves was isolated using Trizol reagent (Invitrogen, Shanghai, China) and total RNA from flowers, nodules, and pods was isolated by Trizol plants reagent (Transgen Biotech, Beijing), which was optimized for total RNA isolation from plant materials containing abundant polysaccharides and polyphenol. Total RNA was treated with DNaseI (Takara, Dalian, China) to eliminate potential genomic DNA contamination. Reverse transcription products were used for subsequent qRT-PCR analysis under the following condition: $5 \mathrm{~min}$ at $95^{\circ} \mathrm{C}$, followed by 40 cycles of $15 \mathrm{~s}$ at $94^{\circ} \mathrm{C}$ and $1 \mathrm{~min}$ at $60^{\circ} \mathrm{C}$. Primers used for qRT-PCR included LjPSK1-F (GTAGCAC CAGAAGGTCCAAG), LjPSK1-R (TAGATGTAATCCAAGT GGGC), LjPSK2-F (TGGCAACCTTCTTCTTCCTT), LjPSK2-R (GCTGTTCATTTCCTCTTCCA), LjPSK3-F (CATTTGCTCG TCCTACTCCT), LjPSK3-R (ACATTCTTCTGTCCCTTCCA), LjPSK4-F (GAATCGCTGGATGTTGAGGA), LjPSK4-R (GGG TTGTTATGCTTCTGCGT), LjPSK5-F (CTAACCAGTGAACA AGGGAA), LjPSK5-R (TGTAGATGTAGTCCAGGTGAGC), LjTPST-F (TGAAGTGCGCCATTGTGTACAG), and LjTPST-R (GCTCCTCAGTAAGACCAACATATAACAT). For overexpression assays, total RNA was isolated from several independent hairyroot lines or Arabidopsis seedlings and was assigned to realtime RT-PCR or semiquantitative RT-PCR, using qRT-PCR primers and AtPSKR1-OX primers, respectively. The AtPSKRI transcripts in Arabidopsis AtPSKR1-OX lines were quantitatively measured by using qRT-PCR primers AtPSKR1-qF (AGTCAAT GAGCCATCTCCTG) and AtPSKRl-qR (CCAAACTCCTCC CAAATAGG). The expression of PDF1.2, JAR1, and MYC2 was quantified in three to eight independent hairy-root lines, using the following primers: LjPDF1.2-F (GTGATCAGAGGTGT AAAGCC), $L j P D F 1.2-\mathrm{R}$ (AGTTATCACTGCACTGGAAG), LjJARl-F (GGTGAAGAGTATGAAATTGTTATGACC), LjJARl-R (CCACATCTCCTAGCCTATACCG), LjMYC2-F (CGAAGAACG GCAGCAGTAAC), and LjMYC2-R (AGCTCAGAATCTCAC CGGATT). The expression levels of each gene were normalized with the LjATPase (AW719841) and AtEF1- $a$ reference genes, using the following primers: ATPase-F (CAATGTC GCCAAGGCCCATGGTG), ATPase-R (AACACCACTCTC GATCATTTCTCTG), and AtEF1-a-F (GAGGCAGACTGTT GCAGTCG), AtEF1-a-R (TCACTTCGCACCCTTCTTGA) in L. japonicus and Arabidopsis, respectively.

\section{PSK- $\alpha$ treatment.}

A. thaliana Col-0 seeds were sterilized with $0.1 \%$ active chlorine for $10 \mathrm{~min}$ and were washed with sterilized water five times. The germination of seeds was synchronized at $4^{\circ} \mathrm{C}$ in the dark for 1 day and were then maintained on one-half Murashige Skoog (MS) medium with a 16-h-light and 8-h-dark cycle at $22 \pm 1{ }^{\circ} \mathrm{C}$. Seedlings were grown on solid medium containing $0.8 \%$ agar and different concentrations of PSK- $\alpha$ peptide, which was synthesized, by the Chinese Peptide Company (Hangzhu, China), with purity of $97.6 \%$. Root length was measured after 6 days of growth. L. japonicus seeds were sterilized and synchronized. The swollen seeds were spread on plates containing one-half MS medium. Plates were placed upside down for 30 to $40 \mathrm{~h}$ at $22^{\circ} \mathrm{C}$ in the dark for embryonic root elongation (approximately $1 \mathrm{~cm}$ ). Seeds were then transferred to new one-half MS plates (1.2\% agar, $\mathrm{pH} 6.8$, with or without $1 \mu \mathrm{M}$ PSK- $\alpha$ ) for 5 days for root elongation. At least 25 plants were measured for root length. For nodulation assays, L. japonicus seedlings were transplanted to growth pots and were inoculated with nitrogenfree liquid medium containing both rhizobia (optical density = 0.03 ) and $1 \mu \mathrm{M}$ PSK- $\alpha$ after 3 days of growth. Plants were supplied with half-strength nitrogen-free liquid medium and 
$1 \mu \mathrm{M}$ PSK- $\alpha$ aqueous solution. Nodule numbers were counted 12 days after inoculation with $M$. loti.

\section{Plasmid construction.}

For overexpression, the $L j P S K 1$ cDNA was placed behind an enhanced CaMV $35 \mathrm{~S}$ promoter using primers LjPSK1-F (AACCATGGAATGAACCAATGAAGCAAC) and LjPSK1-R (CCACTAGTTTAGGGCTTATAGTGTTGG). The whole expression unit was then cloned to the KpnI/SacI site of pCAMBIA2301, generating pC2301-d35S-LjPSK1, which contains both GUS and kanamycin selection markers for A. rhizogenesmediated hairy-root transformation and A. tumefaciens-mediated stable transformation. The AtPSKRl cDNA was amplified using primers AtPSKRl-F (AGCTCTAGATGTTCTTGAAATGCGT GTTCATC) and AtPSKRl-R (AATGGCGCGCCCTAGACAT CATCAAGCCAAG). The PCR fragment was cloned to the $X b a \mathrm{I} / A s c \mathrm{I}$ site of pUBGFP or pUBHyg (Maekawa et al. 2008), generating pUBGFP-AtPSKR1 for hairy-root transformation in L. japonicus and pUBHyg-AtPSKRl for stable transformation in Arabidopsis. AtPSKR1 was expressed under the control of a $U b i$ gene promoter. For expression of $\mathrm{LjCCaMK} \mathrm{T}^{T 265 D}, \mathrm{LjCCaMK}$ cDNA was amplified and cloned to the $X b a \mathrm{I} / A s c \mathrm{I}$ site of pUBGFP using primers $L j C C a M K-F$ (AGCTCTAGAGCCACC ATGGGATATGATCAAACCAGAAAG) and $L j C C a M K-R$ (AA AGGCGCGCCCTATGATGGACGAAGAGAAGAGAG). The T265D site mutation was constructed by primers T265D-mF (GTTTCTATGAGAAGGATTGGAAGGGCATTAC) and T265DmR (GTAATGCCCTTCCAATCCTTCTCATAGAAAC), using an overlapping PCR method. For promoter-GUS reporter analysis, a 4,144-bp promoter fragment upstream of the translation start site (ATG) of LjPSK1 was amplified from the MG20 genomic DNA and was inserted upstream of the GUS gene in the binary vector pX2181 at the BamHI/SmaI site. The promoter fragment was amplified using primers $L j P S K 1 p$-F (AAGGATCCATGG CACCATATACTTGGGC) and LjPSK1p-R (AACCCGGGTG GTTCATTCAGGGAATAAGGG).

\section{Plant transformation.}

Agrobacterium rhizogenes-mediated hairy-root transformation was performed as described previously (Wang et al. 2013). After the hairy roots were generated, positive roots expressing pUBGFP-AtPSKR or pUBGFP were selected using the GFP marker under a fluorescence microscope. For selection of positive hairy roots expressing pC2301-d35S-LjPSK1 or pC2301, root tips of 2 to $3 \mathrm{~mm}$ long were excised for GUS staining. Two GFP-positive or GUS-positive hairy roots were preserved on each plant and all the negative roots were removed. Plants were then transplanted to growth cabinets for 6 days of growth before inoculation with M. loti. Arabidopsis transformation was carried out by the floral-dip method (Clough and Bent 1998). Transgenic seeds were selected on one-half MS plates containing $50 \mathrm{mg}$ of kanamycin or $20 \mathrm{mg}$ of hygromycin per liter.

\section{GUS staining.}

Transgenic hairy roots expressing the GUS reporter gene under the control of the LjPSK1 promoter were inoculated with $M$. loti to induce nodulation. Two weeks later, nodules were washed with water and were incubated in GUS staining solution $\left(100 \mathrm{mM} \mathrm{Na}_{3} \mathrm{PO}_{4}\right.$ buffer, $\mathrm{pH} 7.0,0.1 \%$ Triton $\mathrm{X}-100,0.1 \%$ N-laurylsarcosine, $10 \mathrm{mM}$ EDTA, $1 \mathrm{mM} \mathrm{K}{ }_{3} \mathrm{Fe}(\mathrm{CN})_{6}, 1 \mathrm{mM}$ $\mathrm{K}_{4} \mathrm{Fe}(\mathrm{CN})_{6}$, and $\mathrm{X}-$ Gluc at $\left.0.5 \mathrm{mg} / \mathrm{ml}\right)$ for $8 \mathrm{~h}$ at $37^{\circ} \mathrm{C}$, after vacuuming for $5 \mathrm{~min}$.

\section{IT assay.}

Transgenic plants were grown in growth cabinets for 6 days before inoculation with $M$. loti MAFF303099 harboring a plasmid, pPN28, for constitutive expression of $\beta$-galactosidase (lacZ). IT in hairy roots were visualized after staining with X-gal solution, as described previously (Wang et al. 2013).

\section{Identification of L. japonicus LORE1 mutants.}

Genomic DNAs were isolated from individual mutant plants and were used for PCR amplification of $5 \mathrm{~min}$ at $95^{\circ} \mathrm{C}$ and 35 cycles of $30 \mathrm{~s}$ at $94^{\circ} \mathrm{C}, 30 \mathrm{~s}$ at $60^{\circ} \mathrm{C}$, and $45 \mathrm{~s}$ at $72^{\circ} \mathrm{C}$, followed by $6 \mathrm{~min}$ at $72^{\circ} \mathrm{C}$. Primers for the identification of Ljpsk1 and Ljpsk4 mutants were LjPSK1-mF (TGCTCTGCATTTGA ACTAAGGATGCAC), LjPSK1-mR (TGCTCGTCTCCATGTAG CACCAGAAGG), LjPSK4-mF (TCTGGAGGCCTGGGCCAAG TAACC), and LjPSK4-mR (CCACCTTCCAAGTTTCACACCT CCTCC). Primer P2 (CCATGGCGGTTCCGTGAATCTTAGG) coupled with LjPSK1-mF or LjPSK4-mF were used for the identification of LORE1 insertions as described (Urbański et al. 2012).

\section{DNA sequence analysis.}

Multiple sequence alignment of PSK was performed using ClustalX. The gene structure of LjPSK was predicted by the Spidey program. Sequence data from this article have been deposited to GenBank with accession numbers of KM065832 for LjPSK1, KM065833 for LjPSK2, KM065834 for LjPSK3, KM065835 for LjPSK4, and KM065836 for LjPSK5.

\section{ACKNOWLEDGMENTS}

We thank the Centre for Carbohydrate Recognition and Signaling (CARB) for providing LORE1 mutant seeds (seed ID: 30030761 and 30006132). This work was supported by the National Basic Research Program of China (2011CB100702), the National Natural Science Foundation of China (31370277), Shanghai Key Projects of Basic Research (14JC1402300), and Shanghai Sailing Program (15YF1403800).

\section{LITERATURE CITED}

Breakspear, A., Liu, C., Roy, S., Stacey, N., Rogers, C., Trick, M., Morieri, G., Mysore, K. S., Wen, J., Oldroyd, G. E., Downie, J. A., and Murray, J. D. 2014. The root hair "infectome" of Medicago truncatula uncovers changes in cell cycle genes and reveals a requirement for auxin signaling in rhizobial infection. Plant Cell 26:4680-4701.

Clough, S. J., and Bent, A. F. 1998. Floral dip: A simplified method for Agrobacterium-mediated transformation of Arabidopsis thaliana. Plant J. 16:735-743.

Delay, C., Imin, N., and Djordjevic, M. A. 2013. Regulation of Arabidopsis root development by small signaling peptides. Front. Plant Sci. 4:352.

Downie, J. A. 2014. Legume nodulation. Curr. Biol. 24:R184-R190.

Fang, Y., and Hirsch, A. M. 1998. Studying early nodulin gene ENOD40 expression and induction by nodulation factor and cytokinin in transgenic alfalfa. Plant Physiol. 116:53-68.

Farkas, A., Maróti, G., Durgő, H., Györgypál, Z., Lima, R. M., Medzihradszky, K. F., Kereszt, A., Mergaert, P., and Kondorosi, É. 2014. Medicago truncatula symbiotic peptide NCR247 contributes to bacteroid differentiation through multiple mechanisms. Proc. Natl. Acad. Sci. U.S.A. 111:5183-5188.

Ferguson, B. J., Indrasumunar, A., Hayashi, S., Lin, M. H., Lin, Y. H., Reid, D. E., and Gresshoff, P. M. 2010. Molecular analysis of legume nodule development and autoregulation. J. Integr. Plant Biol. 52:61-76.

Funayama-Noguchi, S., Noguchi, K., Yoshida, C., and Kawaguchi, M. 2011. Two CLE genes are induced by phosphate in roots of Lotus japonicus. J. Plant Res. 124:155-163.

Gleason, C., Chaudhuri, S., Yang, T., Muñoz, A., Poovaiah, B. W., and Oldroyd, G. E. 2006. Nodulation independent of rhizobia induced by a calcium-activated kinase lacking autoinhibition. Nature 441:1149-1152.

Hartmann, J., Stührwohldt, N., Dahlke, R. I., and Sauter, M. 2013. Phytosulfokine control of growth occurs in the epidermis, is likely to be non-cell autonomous and is dependent on brassinosteroids. Plant J. 73:579-590.

Huang, J. Y., Wang, Y. F., and Yang, J. S. 2010. [Over-expression of OsPSK3 increases chlorophyll content of leaves in rice]. Yi Chuan 32: 1281-1289.

Igarashi, D., Tsuda, K., and Katagiri, F. 2012. The peptide growth factor, phytosulfokine, attenuates pattern-triggered immunity. Plant J. 71: 194-204. 
Imin, N., Mohd-Radzman, N. A., Ogilvie, H. A., and Djordjevic, M. A. 2013. The peptide-encoding CEP1 gene modulates lateral root and nodule numbers in Medicago truncatula. J. Exp. Bot. 64:5395-5409.

Komori, R., Amano, Y., Ogawa-Ohnishi, M., and Matsubayashi, Y. 2009. Identification of tyrosylprotein sulfotransferase in Arabidopsis. Proc. Natl. Acad. Sci. U.S.A. 106:15067-15072.

Krusell, L., Madsen, L. H., Sato, S., Aubert, G., Genua, A., Szczyglowski, K., Duc, G., Kaneko, T., Tabata, S., de Bruijn, F., Pajuelo, E., Sandal, N., and Stougaard, J. 2002. Shoot control of root development and nodulation is mediated by a receptor-like kinase. Nature 420:422-426.

Kumagai, H., Kinoshita, E., Ridge, R. W., and Kouchi, H. 2006. RNAi knock-down of ENOD40s leads to significant suppression of nodule formation in Lotus japonicus. Plant Cell Physiol. 47:1102-1111.

Kumagai, H., and Kouchi, H. 2003. Gene silencing by expression of hairpin RNA in Lotus japonicus roots and root nodules. Mol. Plant-Microbe Interact. 16:663-668.

Kutschmar, A., Rzewuski, G., Stührwohldt, N., Beemster, G. T., Inzé, D., and Sauter, M. 2009. PSK- $\alpha$ promotes root growth in Arabidopsis. New Phytol. 181:820-831.

Limpens, E., Franken, C., Smit, P., Willemse, J., Bisseling, T., and Geurts, R. 2003. LysM domain receptor kinases regulating rhizobial Nod factorinduced infection. Science 302:630-633.

Loivamäki, M., Stührwohldt, N., Deeken, R., Steffens, B., Roitsch, T., Hedrich, R., and Sauter, M. 2010. A role for PSK signaling in wounding and microbial interactions in Arabidopsis. Physiol. Plant. 139:348-357.

Madsen, E. B., Madsen, L. H., Radutoiu, S., Olbryt, M., Rakwalska, M., Szczyglowski, K., Sato, S., Kaneko, T., Tabata, S., Sandal, N., and Stougaard, J. 2003. A receptor kinase gene of the LysM type is involved in legume perception of rhizobial signals. Nature 425:637-640.

Maekawa, T., Kusakabe, M., Shimoda, Y., Sato, S., Tabata, S., Murooka, Y., and Hayashi, M. 2008. Polyubiquitin promoter-based binary vectors for overexpression and gene silencing in Lotus japonicus. Mol. PlantMicrobe Interact. 21:375-382.

Matsubayashi, Y., Ogawa, M., Kihara, H., Niwa, M., and Sakagami, Y. 2006. Disruption and overexpression of Arabidopsis phytosulfokine receptor gene affects cellular longevity and potential for growth. Plant Physiol. 142:45-53.

Matsubayashi, Y., and Sakagami, Y. 1996. Phytosulfokine, sulfated peptides that induce the proliferation of single mesophyll cells of Asparagus officinalis L. Proc. Natl. Acad. Sci. U.S.A. 93:7623-7627.

Matsubayashi, Y., Takagi, L., and Sakagami, Y. 1997. Phytosulfokinealpha, a sulfated pentapeptide, stimulates the proliferation of rice cells by means of specific high- and low-affinity binding sites. Proc. Natl. Acad. Sci. U.S.A. 94:13357-13362.

Matsuzaki, Y., Ogawa-Ohnishi, M., Mori, A., and Matsubayashi, Y. 2010. Secreted peptide signals required for maintenance of root stem cell niche in Arabidopsis. Science 329:1065-1067.

Mortier, V., De Wever, E., Vuylsteke, M., Holsters, M., and Goormachtig, S. 2012. Nodule numbers are governed by interaction between CLE peptides and cytokinin signaling. Plant J. 70:367-376.

Mortier, V., Den Herder, G., Whitford, R., Van de Velde, W., Rombauts, S., D'Haeseleer, K., Holsters, M., and Goormachtig, S. 2010. CLE peptides control Medicago truncatula nodulation locally and systemically. Plant Physiol. 153:222-237.

Mortier, V., Fenta, B. A., Martens, C., Rombauts, S., Holsters, M., Kunert, K., and Goormachtig, S. 2011. Search for nodulation-related CLE genes in the genome of Glycine max. J. Exp. Bot. 62:2571-2583.

Mosher, S., Seybold, H., Rodriguez, P., Stahl, M., Davies, K. A., Dayaratne, S., Morillo, S. A., Wierzba, M., Favery, B., Keller, H., Tax, F. E., and Kemmerling, B. 2013. The tyrosine-sulfated peptide receptors PSKR1 and PSY1R modify the immunity of Arabidopsis to biotrophic and necrotrophic pathogens in an antagonistic manner. Plant J. 73:469-482.

Nakagawa, T., and Kawaguchi, M. 2006. Shoot-applied MeJA suppresses root nodulation in Lotus japonicus. Plant Cell Physiol. 47:176-180.

Okamoto, S., Ohnishi, E., Sato, S., Takahashi, H., Nakazono, M., Tabata, S., and Kawaguchi, M. 2009. Nod factor/nitrate-induced CLE genes that drive HAR1-mediated systemic regulation of nodulation. Plant Cell Physiol. 50:67-77.

Okamoto, S., Shinohara, H., Mori, T., Matsubayashi, Y., and Kawaguchi, M. 2013. Root-derived CLE glycopeptides control nodulation by direct binding to HAR1 receptor kinase. Nat. Commun. 4:2191.

Oldroyd, G. E., and Downie, J. A. 2008. Coordinating nodule morphogenesis with rhizobial infection in legumes. Annu. Rev. Plant Biol. 59:519-546.

Penterman, J., Abo, R. P., De Nisco, N. J., Arnold, M. F., Longhi, R., Zanda, M., and Walker, G. C. 2014. Host plant peptides elicit a transcriptional response to control the Sinorhizobium meliloti cell cycle during symbiosis. Proc. Natl. Acad. Sci. U.S.A. 111:3561-3566.
Radutoiu, S., Madsen, L. H., Madsen, E. B., Felle, H. H., Umehara, Y., Grønlund, M., Sato, S., Nakamura, Y., Tabata, S., Sandal, N., and Stougaard, J. 2003. Plant recognition of symbiotic bacteria requires two LysM receptor-like kinases. Nature 425:585-592.

Reid, D. E., Ferguson, B. J., and Gresshoff, P. M. 2011. Inoculation- and nitrate-induced CLE peptides of soybean control NARK-dependent nodule formation. Mol. Plant-Microbe Interact. 24:606-618.

Roux, B., Rodde, N., Jardinaud, M. F., Timmers, T., Sauviac, L., Cottret, L., Carrère, S., Sallet, E., Courcelle, E., Moreau, S., Debellé, F., Capela, D., de Carvalho-Niebel, F., Gouzy, J., Bruand, C., and Gamas, P. 2014. An integrated analysis of plant and bacterial gene expression in symbiotic root nodules using laser-capture microdissection coupled to RNA sequencing. Plant J. 77:817-837.

Schnabel, E., Journet, E.-P., de Carvalho-Niebel, F., Duc, G., and Frugoli, J. 2005. The Medicago truncatula SUNN gene encodes a $C_{L V}$-like leucine-rich repeat receptor kinase that regulates nodule number and root length. Plant Mol. Biol. 58:809-822.

Searle, I. R., Men, A. E., Laniya, T. S., Buzas, D. M., Iturbe-Ormaetxe, I., Carroll, B. J., and Gresshoff, P. M. 2003. Long-distance signaling in nodulation directed by a CLAVATA1-like receptor kinase. Science 299: 109-112.

Smit, P., Limpens, E., Geurts, R., Fedorova, E., Dolgikh, E., Gough, C., and Bisseling, T. 2007. Medicago LYK3, an entry receptor in rhizobial nodulation factor signaling. Plant Physiol. 145:183-191.

Srivastava, R., Liu, J. X., and Howell, S. H. 2008. Proteolytic processing of a precursor protein for a growth-promoting peptide by a subtilisin serine protease in Arabidopsis. Plant J. 56:219-227.

Stührwohldt, N., Dahlke, R. I., Steffens, B., Johnson, A., and Sauter, M. 2011. Phytosulfokine- $\alpha$ controls hypocotyl length and cell expansion in Arabidopsis thaliana through phytosulfokine receptor 1. PLoS ONE 6:e21054.

Sun, J., Cardoza, V., Mitchell, D. M., Bright, L., Oldroyd, G., and Harris, J. M. 2006. Crosstalk between jasmonic acid, ethylene and Nod factor signaling allows integration of diverse inputs for regulation of nodulation. Plant J. 46:961-970.

Suzuki, A., Suriyagoda, L., Shigeyama, T., Tominaga, A., Sasaki, M., Hiratsuka, Y., Yoshinaga, A., Arima, S., Agarie, S., Sakai, T., Inada, S. Jikumaru, Y., Kamiya, Y., Uchiumi, T., Abe, M., Hashiguchi, M., Akashi, R., Sato, S., Kaneko, T., Tabata, S., and Hirsch, A. M. 2011. Lotus japonicus nodulation is photomorphogenetically controlled by sensing the red/far red (R/FR) ratio through jasmonic acid (JA) signaling Proc. Natl. Acad. Sci. U.S.A. 108:16837-16842.

Tirichine, L., Imaizumi-Anraku, H., Yoshida, S., Murakami, Y., Madsen, L. H., Miwa, H., Nakagawa, T., Sandal, N., Albrektsen, A. S., Kawaguchi, M., Downie, A., Sato, S., Tabata, S., Kouchi, H., Parniske, M., Kawasaki, S., and Stougaard, J. 2006. Deregulation of a $\mathrm{Ca}_{2}^{+} /$calmodulin-dependent kinase leads to spontaneous nodule development. Nature 441:1153-1156.

Urbański, D. F., Małolepszy, A., Stougaard, J., and Andersen, S. U. 2012. Genome-wide LORE1 retrotransposon mutagenesis and high-throughput insertion detection in Lotus japonicus. Plant J. 69:731-741.

Van de Velde, W., Zehirov, G., Szatmari, A., Debreczeny, M., Ishihara, H., Kevei, Z., Farkas, A., Mikulass, K., Nagy, A., Tiricz, H., SatiatJeunemaître, B., Alunni, B., Bourge, M., Kucho, K., Abe, M., Kereszt, A., Maroti, G., Uchiumi, T., Kondorosi, E., and Mergaert, P. 2010. Plant peptides govern terminal differentiation of bacteria in symbiosis. Science 327:1122-1126.

Wan, X., Hontelez, J., Lillo, A., Guarnerio, C., van de Peut, D., Fedorova, E., Bisseling, T., and Franssen, H. 2007. Medicago truncatula ENOD40-1 and ENOD40-2 are both involved in nodule initiation and bacteroid development. J. Exp. Bot. 58:2033-2041.

Wang, C., Zhu, H., Jin, L., Chen, T., Wang, L., Kang, H., Hong, Z., and Zhang, Z. 2013. Splice variants of the SIP1 transcripts play a role in nodule organogenesis in Lotus japonicus. Plant Mol. Biol. 82: 97-111.

Yang, H., Matsubayashi, Y., Hanai, H., Nakamura, K., and Sakagami, Y. 2000. Molecular cloning and characterization of OsPSK, a gene encoding a precursor for phytosulfokine- $\alpha$, required for rice cell proliferation. Plant Mol. Biol. 44:635-647.

Yang, H., Matsubayashi, Y., Nakamura, K., and Sakagami, Y. 2001. Diversity of Arabidopsis genes encoding precursors for phytosulfokine, a peptide growth factor. Plant Physiol. 127:842-851.

\section{AUTHOR-RECOMMENDED INTERNET RESOURCE}

The Spidey program: http://www.ncbi.nlm.nih.gov/spidey 